QUARTERLY OF APPLIED MATHEMATICS

VOLUME LXVII, NUMBER 4

DECEMBER 2009, PAGES 745-754

S 0033-569X(09)01158-2

Article electronically published on May 14, 2009

\title{
MULTIPLICATIVE FINITE DIFFERENCE METHODS
}

\author{
BY \\ MUSTAFA RIZA (Department of Mathematics, Eastern Mediterranean University, Gazimağusa - \\ North Cyprus, via Mersin 10, Turkey), \\ ALI ÖZYAPICI (Department of Mathematics, Ege University, Bornova, Izmir, Turkey), \\ AND \\ EMINE MISIRLI (Department of Mathematics, Ege University, Bornova, Izmir, Turkey)
}

\begin{abstract}
Based on multiplicative calculus, the finite difference schemes for the numerical solution of multiplicative differential equations and Volterra differential equations are presented. Sample problems were solved using these new approaches.
\end{abstract}

1. Introduction. Michael Grossman and Robert Katz have indicated in [1] that infinitely many calculi can be constructed independently. Each of these calculi provide different perspectives for approaching many problems in science and engineering. Additionally, a mathematical problem which is difficult or impossible to solve in one calculus can be easily revealed through another calculus. E.g. the Volterra calculus [2], called after Vito Volterra, was introduced to define the derivative of dimensional functions that could not be done using the derivative in the Newtonian sense. Independently, Grossmann introduced bigeometric calculus [3] 45 years later, which turned out to be identical to Volterra calculus. These works stimulate the idea that it can be useful to generate a new calculus according to the area of study. With respect to this idea, it seems to be evident that multiplicative and Volterra differential calculus can be used more effectively as a mathematical tool instead of ordinary differential calculus for the mathematical representation of many problems in science and engineering that can be easily represented in these calculi. Indeed, problems related to growth rates can be expressed effectively within the framework of multiplicative calculus [4. Additionally, recent studies [6, 7] show the importance of usage of Volterra differential calculus in mathematical modeling.

Received August 24, 2008.

2000 Mathematics Subject Classification. Primary 65L12; Secondary 65N06.

Key words and phrases. Finite difference method, multiplicative calculus, Volterra calculus, bigeometric calculus.

The first author was supported by the A-Type Research Grant of Eastern Mediterranean University.

E-mail address: mustafa.riza@emu.edu.tr

E-mail address: ali.ozyapici@emu.edu.tr

E-mail address: emine.kurpinar@ege.edu.tr 
After the usage of multiplicative and Volterra differential calculus, their respective solutions have been becoming more and more important and inevitable. Considering that the exact solutions of desired differential equations cannot always be obtained in closed form, numerical approximations of multiplicative differential equations become necessary. Recently the multiplicative Runge-Kutta Method for Volterra differential equations was discussed in [6]. In analogy to numerical solutions of Newtonian differential equations, the multiplicative finite difference scheme is introduced in this article for the numerical solution of multiplicative differential equations. The Volterra finite difference scheme is represented in the framework of the multiplicative finite difference scheme.

\section{Multiplicative and Volterra differential equations.}

2.1. Multiplicative differentiation and differential equations. Let $f$ with respect $x$ to be a real positive valued function defined in open interval $(a, b)$. Suppose that the value changes from $x$ to $x+h$ in the given interval. Then, the function $f$ changes from $f(x)$ to $f(x+h)$. Consequently, a new operator $\Delta^{*}$ in the multiplicative sense can be defined over the change of $f$ such that

$$
\Delta^{*} f(x)=\frac{f(x+h)}{f(x)} .
$$

Using operator (2.1), a new kind of definition of derivative can be given as follows:

Definition 2.1. Let $f$ be a function with respect to $x$, where $x \in \operatorname{dom}(f)$. If the limit

$$
f^{*}(x)=\lim _{h \rightarrow 0}\left(\Delta^{*} f\right)^{\frac{1}{h}}=\lim _{h \rightarrow 0}\left(\frac{f(x+h)}{f(x)}\right)^{\frac{1}{h}}
$$

exists, then $f$ is called the multiplicative differentiable at $x$.

If $f$ is a positive function and the derivative of $f$ at $x$ exists, then the $n^{\text {th }}$ multiplicative derivative of $f$ exists and

$$
f^{*(n)}(x)=\exp \left\{(\ln \circ f)^{(n)}(x)\right\} .
$$

Some properties of the multiplicative derivative are stated by the following theorem [4.

TheOREm 2.2. Let $f$ and $g$ be differentiable in the multiplicative sense. Then, $c f, f \cdot g$, $f+g, f / g$, and $f^{h}$ are differentiable in the multiplicative sense, and

$$
\begin{aligned}
(c f)^{*}(t) & =f^{*}(t), \\
(f \cdot g)^{*}(t) & =f^{*}(t) g^{*}(t), \\
(f+g)^{*}(t) & =f^{*}(t)^{\frac{f(t)}{f(t)+g(t)}} \cdot g^{*}(t)^{\frac{g(t)}{f(t)+g(t)}}, \\
(f / g)^{*}(t) & =f^{*}(t) / g^{*}(t), \\
\left(f^{h}\right)^{*}(x) & =f^{*}(x)^{h(x)} \cdot f(x)^{h^{\prime}(x)},
\end{aligned}
$$

where $c$ is a positive constant, $f$ and $g$ are $*$-differentiable, and $h$ is differentiable.

Thus, the multiplicative derivative is considerably different from the ordinary derivative. Indeed, the formulae for finding the derivative of multiplication or division of two functions are easy in multiplicative calculus. 
It is reasonable to consider in the next step the differential equations involving *derivatives. The equation

$$
y^{*}(x)=f(x, y(x))
$$

containing the $*$-derivative of $y$ is called multiplicative differential equation. This equation makes sense if $f$ is a positive function defined in some subset $G$ of $\mathbb{R} \times \mathbb{R}^{+}$. The existence and uniqueness of the solutions is shown in 4 .

Specifically, the solutions of the simplest case of multiplicative differential equations of first and second order, namely

$$
y^{*}=c \quad \text { and } \quad y^{* *}=c,
$$

can be given as

$$
y=\alpha c^{x} \quad \text { and } \quad y=\alpha c^{\frac{x^{2}}{2}+\beta x}
$$

respectively, where $\alpha>0$ and $\beta$ are constants.

2.2. Volterra differentiation and differential equations. Another type of multiplicative calculus, with its main operator being multiplication, was created by Volterra [2], and a new kind of derivative was given within this calculus. To prevent any conflict, we refer to this type of multiplicative calculus as Volterra calculus. For more details, the interested reader is referred to [5, 6, 7]. The definition of this kind of derivative can be given as follows.

Definition 2.3. Let $f$ be a positive function over the open interval $(a, b)$. If the limit

$$
f^{\pi}(x)=\frac{d^{\pi} f(x)}{d x}=\lim _{h \rightarrow 0}\left(\frac{f((1+h) x)}{f(x)}\right)^{\frac{1}{h}}
$$

exists, then $f$ is said to be Volterra type differentiable at $x \in(a, b)$.

The relationship between the ordinary and the Volterra derivative can be given as

$$
f^{\pi}(x)=\exp \left(x(\ln \circ f)^{\prime}(x)\right) .
$$

Consequently, by using (2.3) the Volterra derivative can be written as a multiplicative derivative as follows:

$$
f^{\pi}(x)=\frac{d^{\pi} f(x)}{d x}=\left(f^{*}(x)\right)^{x} .
$$

The representation of higher order Volterra derivatives in terms of the ordinary derivatives is complicated. As the relationship between the Volterra derivative and the multiplicative derivative looks very simple, the idea of representing the Volterra derivatives in terms of the multiplicative derivatives seems to be straightforward.

E.g. Using (2.8) and (2.12) the Volterra derivatives of order 2-4 can be given in terms of the multiplicative derivative as

$$
\begin{aligned}
f^{\pi \pi}(x) & =\left(f^{* *}(x)\right)^{x^{2}}\left(f^{*}\right)^{x}, \\
f^{\pi(3)}(x) & =\left(f^{*(3)}(x)\right)^{x^{3}}\left(f^{* *}(x)\right)^{3 x^{2}}\left(f^{*}\right)^{x}, \\
f^{\pi(4)}(x) & =\left(f^{*(4)}(x)\right)^{x^{4}}\left(f^{*(3)}(x)\right)^{6 x^{3}}\left(f^{* *}(x)\right)^{7 x^{2}}\left(f^{*}\right)^{x} .
\end{aligned}
$$


Analogously to the multiplicative differential equations, it is reasonable to consider in the next step the differential equations involving $\pi$-derivatives. The equation

$$
y^{\pi}(x)=f(x, y(x))
$$

containing the $\pi$-derivative of $y$ is called a Volterra differential equation. This equation makes sense if $f$ is a positive function defined in some subset $G$ of $\mathbb{R}^{+} \times \mathbb{R}^{+}$. The existence and uniqueness of the solutions can be seen following the results of the multiplicative differential equations as shown in 4 .

\section{Finite difference methods.}

3.1. Derivation of the multiplicative finite difference method. The multiplicative Taylor theorem is the starting point for the derivation of the multiplicative finite difference scheme. According to [4] the multiplicative Taylor theorem is given as:

Theorem 3.1. (Multiplicative Taylor theorem). Let $f$ be a $(n+1)$-times *-differentiable function on $(a, b)$. Assume that $x_{0} \in[a, b]$. Then, for every $x$ in $[a, b], x \neq x_{0}$, there exists a point $x_{1}$ between $x$ and $x_{0}$ such that

$$
f(x)=f\left(x_{0}\right) \prod_{k=1}^{n}\left(f^{*(k)}\left(x_{0}\right)\right)^{\frac{\left(x-x_{0} k\right.}{k !}}\left(f^{*(n+1)}\left(x_{1}\right)\right)^{\frac{\left(x-x_{0}\right)^{n+1}}{(n+1) !}} .
$$

From (3.1) the multiplicative Taylor expansion of $f(x+h)$ is then

$$
f(x+h)=\prod_{n=0}^{\infty}\left[f^{*(n)}(x)\right]^{\frac{h^{n}}{n !}} .
$$

From (3.2) the first four terms of forward $(+)$ and backward (-) expansion with $h$ can be given respectively:

$$
f(x \pm h)=f(x)\left[f^{*}(x)\right]^{ \pm h}\left[f^{* *}(x)\right]^{\frac{h^{2}}{2 !}}\left[f^{*(3)}(x)\right]^{ \pm \frac{h^{3}}{3 !}}\left[f^{*(4)}(x)\right]^{\frac{h^{4}}{4 !}} \cdot \ldots \quad .
$$

The approximation of the first order multiplicative derivative can be obtained by dividing the forward expansion by the backward expansion and reordering of the terms. Therefore we get the approximation for the first multiplicative derivative as

$$
f^{*}(x)=\left(\frac{f(x+h)}{f(x-h)}\right)^{\frac{1}{2 h}} \cdot \prod_{n=1}^{\infty}\left(f^{*(2 n+1)}(x)\right)^{-\frac{h^{2 n}}{(2 n+1) !}} .
$$

Analogously we can obtain the approximation of the second order multiplicative derivative by multiplying the forward and the backward expansion yielding to

$$
f^{* *}(x)=\left(\frac{f(x+h) f(x-h)}{f(x)^{2}}\right)^{\frac{1}{h^{2}}} \cdot \prod_{n=1}^{\infty}\left(f^{*(2 n+2)}(x)\right)^{-\frac{2 h^{2 n}}{(2 n+2) !}} .
$$

Omitting the remainder terms of (3.4) and (3.5) we can now derive the multiplicative finite difference method for second order multiplicative differential equation of the form:

$$
f^{* *}(x)=g\left(x, f, f^{*}\right), f(a)=\alpha, f(b)=\beta
$$


Let the interval $[a, b]$ be partitioned by points $a=x_{0}, x_{1}, x_{2}, \ldots, x_{n+1}=b$. These need not to be equally spaced, but for simplicity we assume

$$
x_{i}=a+i h, \quad 0 \leq i \leq n+1, \quad h=\frac{b-a}{n+1}
$$

The discrete version of (3.6) is:

$$
\begin{aligned}
{\left[\frac{f_{i+1} f_{i-1}}{f_{i}^{2}}\right]^{\frac{1}{h^{2}}} } & =g\left(x_{i}, f_{i},\left(\frac{f_{i+1}}{f_{i-1}}\right)^{1 / 2 h}\right) \\
f_{0} & =\alpha \\
f_{n+1} & =\beta .
\end{aligned}
$$

This method can be simplified by taking the natural logarithm:

$$
\begin{aligned}
\frac{1}{h^{2}}\left[k_{i+1}+k_{i-1}-2 k_{i}\right] & =\phi\left(x_{i}, k_{i}, \frac{1}{2 h}\left(k_{i+1}-k_{i-1}\right)\right) \\
k_{0} & =\ln \alpha \\
k_{n+1} & =\ln \beta .
\end{aligned}
$$

where

$$
k_{i}=\ln f_{i} \quad \text { and } \quad \phi\left(x_{i}, k_{i}, \frac{1}{2 h}\left(k_{i+1}-k_{i-1}\right)\right)=\ln \left[g\left(x_{i}, f_{i},\left(\frac{f_{i+1}}{f_{i-1}}\right)^{1 / 2 h}\right)\right]
$$

3.2. Error estimation for the multiplicative finite difference method. If we assume that the main contribution to the error comes from the lowest order multiplicative derivative in the error term, we can approximate the error of the first multiplicative derivative as:

$$
E\left(f^{*}(x)\right) \approx\left(\left|f^{*(3)}(x)\right|^{*}\right)^{-\frac{h^{2}}{3 !}}
$$

where $|\cdot|^{*}$ represents the multiplicative absolute value function given as

$$
|x|^{*}= \begin{cases}x & \text { for } x \geq 1 \\ \frac{1}{x} & \text { for } x<1\end{cases}
$$

Analogously we can apply the same idea to the approximation of the second order multiplicative derivative, and the error yields to:

$$
E\left(f^{* *}(x)\right) \approx\left(\left|f^{*(4)}(x)\right|^{*}\right)^{-\frac{h^{2}}{12}} .
$$

As mentioned above, $f(x)$ must be a positive function. A possible positive function is the exponential function. Recalling the relation between the multiplicative derivative and the ordinary derivative (2.3) 4], and recalling that the invariant function under the multiplicative derivative is $\exp (\exp (x))$, it seems evident to make the ansatz $f(x)=$ $\exp (y(x))$, where $y(x)$ is a real-valued function. Then we can write the multiplicative derivatives of $f$ easily in terms of ordinary derivatives of $y$ as

$$
f^{*(n)}(x)=\exp \left\{(\ln \circ \exp (y(x)))^{(n)}(x)\right\}=\exp \left\{y(x)^{(n)}(x)\right\} .
$$


The errors for $f^{*}(x)$ and $f^{* *}(x)$ simplify under this ansatz to

$$
\begin{aligned}
& E\left(f^{*}(x)\right) \approx\left(f^{*(3)}(x)\right)^{-\frac{h^{2}}{3 !}}=\exp \left\{-\frac{h^{2}}{3 !} y^{(3)}(x)\right\}, \\
& E\left(f^{* *}(x)\right) \approx\left(f^{*(4)}(x)\right)^{-\frac{h^{2}}{12}}=\exp \left\{-\frac{h^{2}}{12} y^{(4)}(x)\right\} .
\end{aligned}
$$

Obviously if $y(x)$ is a polynomial $P_{n}(x)=\sum_{i=0}^{n} a_{i} x^{i}$ of degree $n$, we get an exact approximation for $n<3$ for the first derivative and $n<4$ for the second multiplicative derivative. The example discussed in section 4.1 complies with this statement.

3.3. Derivation of the Volterra finite difference method. Considering the fact that the Volterra Taylor theorem has not been found yet, the Volterra finite difference scheme cannot be derived directly. The multiplicative finite difference method can be used to construct a finite difference method for the approximation of Volterra differential equations. Consequently, the first and second order $\pi$-derivatives can be approximated by using the approximation of the respective multiplicative derivatives derived in section 3.1 .

$$
\begin{aligned}
f^{\pi}(x) & =\left(\frac{f(x+h)}{f(x-h)}\right)^{\frac{x}{2 h}}, \\
f^{\pi \pi}(x) & =\left(\frac{f(x+h) f(x-h)}{f(x)^{2}}\right)^{\frac{x^{2}}{h^{2}}}\left(\frac{f(x+h)}{f(x-h)}\right)^{\frac{x}{2 h}} .
\end{aligned}
$$

Analogously to the multiplicative finite difference method discussed in section 3.1 we can give the discretized version of the first $\pi$-derivative (3.12) and second $\pi$-derivative (3.13) using (2.12) and (2.13). The Volterra finite difference scheme for the Volterra differential equations of the type

$$
f^{\pi \pi}(x)=g\left(x, f, f^{\pi}\right)
$$

with the boundary conditions $f(a)=\alpha$ and $f(b)=\beta$ can be deduced easily from the multiplicative finite difference scheme. Again, the interval $[a, b]$ is divided into $n$ subintervals, and the points of evaluation are given by $x_{i}=a+i h$, with $h=(b-a) /(n+1)$. The finite difference method for the $\pi$-differential equation (3.14) then yields to

$$
\left(\frac{f_{i+1} f_{i-1}}{f_{i}^{2}}\right)^{\frac{x_{i}^{2}}{h^{2}}} \cdot\left(\frac{f_{i+1}}{f_{i-1}}\right)^{\frac{x_{i}}{2 h}}=g\left(x_{i}, f_{i},\left(\frac{f_{i+1}}{f_{i-1}}\right)^{\frac{x_{i}}{2 h}}\right)
$$

with $f_{i}=f\left(x_{i}\right)$. Applying the natural logarithm on both sides we can rewrite equation (3.15) as

$$
\left(\frac{x_{i}^{2}}{h^{2}}+\frac{x_{i}}{2 h}\right) k_{i+1}+\left(\frac{x_{i}^{2}}{h^{2}}-\frac{x_{i}}{2 h}\right) k_{i-1}-\frac{2 x_{i}^{2}}{h^{2}} k_{i}=\phi\left(x_{i}, k_{i}, \frac{x_{i}}{2 h}\left(k_{i+1}-k_{i-1}\right)\right),
$$

where

$$
k_{i}=\ln f_{i}, \quad \phi\left(x_{i}, k_{i}, \frac{x_{i}}{2 h}\left(k_{i+1}-k_{i-1}\right)\right)=\ln \left[g\left(x_{i}, f_{i},\left(\frac{f_{i+1}}{f_{i-1}}\right)^{\frac{x_{i}}{2 h}}\right)\right] .
$$

As the Taylor theorem for Volterra calculus could not yet be obtained, the error cannot be estimated directly. Therefore after conversion of the Volterra type differential equation 
to the multiplicative differential equation an error estimate can be given using the error estimation for the first (3.7) and second (3.9) multiplicative derivatives, discussed in section 3.2 .

4. Applications. This section deals with the application of the newly introduced finite difference methods. The numerical values of the examples are obtained using Mathematica $^{\mathrm{TM}}$.

4.1. Application to ordinary differential equations. Naturally at the beginning we should look at the easiest form of the multiplicative differential equations. The easiest first order multiplicative differential equation $y^{*}(x)=c, c \in \mathbb{R}_{+}$, being a positive real constant, has the trivial solution of the form $y(x)=c^{x}$.

Let us now consider the simplest nontrivial multiplicative differential equation of second order:

$$
y^{* *}(x)=\mathrm{e} .
$$

Using the relation between the multiplicative derivative and the ordinary derivative as given in equation (2.3), we get for the second multiplicative derivative

$$
y^{* *}(x)=\exp \left\{\frac{y^{\prime \prime}(x) y(x)-\left(y^{\prime}(x)\right)^{2}}{y(x)^{2}}\right\} .
$$

Inserting this into (4.1) and taking the logarithm on both sides gives us the ordinary differential equation

$$
y^{\prime \prime}(x)-\frac{y^{\prime}(x)^{2}}{y(x)}-y(x)=0 .
$$

The exact solution of this differential equation can be given as

$$
y(x)=\alpha \exp \left\{\frac{x^{2}}{2}+\beta x\right\},
$$

where $\alpha>0$ and $\beta$ are constants. For the boundary conditions $y(1)=\exp (3 / 2), y(2)=$ $\exp (4)$, i.e. $\alpha=1$ and $\beta=1$, we can see that the multiplicative finite difference method can be used effectively as shown in the table below, whereas the application of the ordinary finite difference method fails extremely.

\begin{tabular}{|l|l|l|l|}
\hline$x$ & $y(x)$ & $y_{\mathrm{a} p p}(x)$ & relative Error in $\%$ \\
\hline 1.25 & 7.62360991 & 7.62360991 & $3.5 \times 10^{-13}$ \\
\hline 1.5 & 13.80457418 & 13.80457418 & $5.3 \times 10^{-13}$ \\
\hline 1.75 & 26.60913187 & 26.60913187 & $1.8 \times 10^{-13}$ \\
\hline
\end{tabular}

This result is in good agreement with the error analysis carried out in section 3.2 . As shown in (3.11) the error for the second derivative is related to derivatives of orders higher than 4. Obviously all multiplicative derivatives of order higher than 3 are equal to 1 , and therefore the calculated error is approximately equal to the machine epsilon $\epsilon_{\text {mach }}$. This means that the multiplicative finite difference method gives the exact solution for this multiplicative differential equation. 
4.2. Application to multiplicative differential equations. Let us consider the following multiplicative differential equation:

$$
y^{* *}=\left(y^{*}\right)^{x}
$$

The analytic solution of this equation is

$$
y(x)=\exp \left\{c_{1}+c_{2} \operatorname{erfi}(x / \sqrt{2})\right\}
$$

where $c_{1}$ and $c_{2}$ are constants.

For the boundary conditions

$$
y(1)=\exp (1.15) \quad \text { and } \quad y(2)=\exp (2)
$$

the constants where chosen to be $c_{1}=0.8625841$ and $c_{2}=0.301452$.

The solution of the multiplicative differential equation (4.4) can be approximated as shown in table below. We can summarize the numerical results for the stepsizes $n=3,7$, and 24 in tabular form as following:

\begin{tabular}{|l|l|l|l|}
\hline \multicolumn{5}{|c|}{$n=3$} \\
\hline$x$ & $y(x)$ & $y_{\text {a } p}(x)$ & relative Error $\%$ \\
\hline 1.25 & 3.53916717 & 3.53731741 & $5.2 \times 10^{-2}$ \\
\hline 1.5 & 4.13636037 & 4.13185178 & $1.1 \times 10^{-1}$ \\
\hline 1.75 & 5.19205181 & 5.18508358 & $1.3 \times 10^{-1}$ \\
\hline \multicolumn{4}{|c|}{$n=7$} \\
\hline 1.125 & 3.32990281 & 3.32969359 & $6.3 \times 10^{-3}$ \\
\hline 1.5 & 4.13636037 & 4.13522500 & $2.7 \times 10^{-2}$ \\
\hline 1.875 & 6.06755685 & 6.06600705 & $2.5 \times 10^{-2}$ \\
\hline \multicolumn{5}{|c|}{$n=24$} \\
\hline 1.04 & 3.20973430 & 3.20972793 & $2.0 \times 10^{-4}$ \\
\hline 1.56 & 4.33342627 & 4.33329159 & $3.1 \times 10^{-3}$ \\
\hline 1.92 & 6.47842153 & 6.47829584 & $1.9 \times 10^{-3}$ \\
\hline
\end{tabular}

The natural logarithm of the results given in table above give the numerical approximation of the ordinary differential equation

$$
k^{\prime \prime}=x k^{\prime} .
$$

4.3. Volterra-type differential equation. Consider the Volterra-type equation

$$
f^{\pi \pi} f^{\pi}=\mathrm{e}^{x} .
$$

The general solution of this equation is

$$
f(x)=\exp \left\{\frac{x}{2}-\frac{c_{1}}{x}+c_{2}\right\} .
$$

For the numerical approximation let us take as an example the constants as $c_{1}=1$ and $c_{2}=0$. For these constants $c_{1}$ and $c_{2}$ equation (4.7) simplifies to

$$
f(x)=\exp \left\{\frac{x}{2}-\frac{1}{x}\right\} .
$$


The approximation for the Volterra-type differential equation (4.6) in the interval $[1,2]$ with the boundary conditions $f(1)=\exp \left\{\frac{1}{2}-1\right\}$ and $f(2)=\exp \left\{1-\frac{1}{2}\right\}$ are given for the step size 3 in the table below:

\begin{tabular}{|l|l|l|l|}
\hline$x$ & $y(x)$ & $y_{\mathrm{a} p p}(x)$ & relative Error in $\%$ \\
\hline 1.25 & 0.839457 & 0.839457 & $1.1 \times 10^{-16}$ \\
\hline 1.5 & 1.0869 & 1.0869 & $1.2 \times 10^{-16}$ \\
\hline 1.75 & 1.35469 & 1.35469 & $1.1 \times 10^{-16}$ \\
\hline
\end{tabular}

As one can see the errors are in the range of the machine epsilon $\epsilon_{m a c h}$, so the approximation seems to be fairly good.

5. Conclusion. A tailor-made calculus can be created for any class of problems. Ergo in the framework of this new calculus, also differential equations used in the mathematical modeling process simplify. Closed form solutions can only be obtained for very few differential equations, hence numerical approximations become inevitable. The easiest approach for constructing numerical approximations in the framework of this new calculus is to derive the finite difference scheme analogously to the finite difference scheme in ordinary (Newtonian) calculus. Naturally, one can expect that the finite difference scheme in the framework of the new calculus will give better approximations for the problems that can be modeled easier in this calculus with less computational effort and higher precision. So, for a certain set of problems this finite difference scheme will be a more efficient and effective numerical approximation.

Multiplicative calculus and Volterra calculus (or bigeometric calculus in Grossman terminology) had been invented a long time ago. Unfortunately, mathematicians did not pay attention to these calculi, although they could be considered as a potential mathematical tool for a certain category of problems. In order to get a good understanding of these calculi and their implications, many studies have to be carried out. In this sense, the multiplicative finite difference scheme, analogous to the well-known finite difference scheme in ordinary calculus, is introduced in this article for numerical approximation of multiplicative and Volterra differential equations. This theory is also applied to sample problems to show their applicability.

6. Acknowledgments. The authors want to thank the Eastern Mediterranean University for funding (A-type research project) the research visit of Dr. M. Riza to Dr. C. Bracher at California State University Long Beach to enable discussions about this topic and on topics related to three-dimensional quantum mechanical tunneling and to start a collaboration on these topics. Also many thanks to Dr. C. Bracher for the valuable discussions how these type of new calculi can be implemented into physics effectively. We would also like to thank T. Karanfiller for his outstanding support and valuable discussions for the determination of the numeric values carried out in section 4 . 


\section{REFERENCES}

[1] M. Grossmann, R. Katz, Non-Newtonian Calculus, Lee Press, Pigeon Cove, Massachusetts, 1972. MR0430173 (55:3180)

[2] V. Volterra, B. Hostinsky, Operations Infinitesimales Lineares, Gauthier-Villars, Paris, 1938.

[3] M. Grossmann, Bigeometric Calculus, A System with a Scale-free Derivative, Archimedes Foundation, Rockport, 1983. MR695495 (84h:26003)

[4] A. Bashirov, E. Kurpınar, A. Ozyapıcı, Multiplicative calculus and its applications, Journal of Mathematical Analysis and Applications 337 (1) (2008) 36-48. MR2356052 (2008k:26001)

[5] M. Ryabczuk, A. Kedzia, W. Zielinski, The concept of physical and fractal dimension II. The differential calculus in dimensional spaces, Chaos Solitons Fractals 12 (2001) 2537-2552. MR1851077

[6] D. Aniszewska, Multiplicative Runge-Kutta methods, Nonlinear Dynamics 50 (1-2) (2007) 265-272.

[7] W. Kasprzak, B. Lysik, M. Rybaczuk, Dimensions, invariants models and fractals, in: Ukranian Society on Fracture Mechanics SPOLOM, Wroclaw-Lviv, Poland, 2004. 